\title{
Orai1 Expression Pattern in Tooth and Craniofacial Ectodermal Tissues and Potential Functions During Ameloblast Differentiation
}

\author{
Li Zheng, ${ }^{1,2}$ Vina Zinn ${ }^{1}$ Anna Lefkelidou, ${ }^{1}$ Nawar Taqi, ${ }^{1}$ Xanthippi Chatzistavrou, ${ }^{1}$ Tarek Balam, ${ }^{1}$ Jeanne Nervina, ${ }^{1}$ \\ Silvana Papagerakis, ${ }^{2,3}$ and Petros Papagerakis ${ }^{1,4,5 *}$ \\ ${ }^{1}$ Department of Orthodontics and Pediatric Dentistry, School of Dentistry, University of Michigan, Ann Arbor, Michigan \\ ${ }^{2}$ Department of Otolaryngology, School of Medicine, University of Michigan, Ann Arbor, Michigan \\ ${ }^{3}$ Department of Periodontology and Oral Medicine, School of Dentistry, University of Michigan, Ann Arbor, Michigan \\ ${ }^{4}$ Center for Organogenesis, School of Medicine, University of Michigan, Ann Arbor, Michigan \\ ${ }^{5}$ Center for Computational Medicine and Bioinformatics, School of Medicine, University of Michigan, Ann Arbor, Michigan
}

Background: Orai1 is a plasma membrane protein that forms the pore of the calcium release activated calcium channel. Humans with mutated Orai1 present with hereditary combined immunodeficiency, congenital myopathy and anhidrotic ectodermal dysplasia. Consistent with the ectodermal dysplasia phenotype, enamel formation and mineralization is also abnormal in Orai1 deficient patients. The expression pattern and potential functions of Orai1 in enamel formation remains unclear. To contribute toward understanding the role of Orai1 in amelogenesis we characterized ORAl1 protein developmental pattern in comparison with other ectodermal organs. We also examined the effects of Orai1 down-regulation in ameloblast cell proliferation and differentiation. Results: Our data show strong expression of ORAl1 protein during the ameloblast secretory stage, which weans at the end of the maturation stage. In salivary glands, ORAI1 is expressed mainly in acini cells. ORAl1 expression is also found in hair follicle and oral epithelium. Knockdown of Orai1 expression decreases cell proliferation and results in RNA expression levels changes of key ameloblast genes regulating enamel thickness and mineralization. Conclusions: This study provides insights in the anhidrotic ectodermal dysplasia phenotype due to Orai1 mutation and highlights the importance of calcium signaling in controlling ameloblast differentiation and maturation during tooth development. Developmental Dynamics 244:1249-1258, 2015. @ 2015 Wiley Periodicals, Inc.

Key words: calcium channels; enamel; dentin; ectodermal dysplasia; amelogenesis imperfect; oral epithelium; salivary glands; hair follicle

Submitted 7 October 2014; First Decision 24 June 2015; Accepted 1 July 2015; Published online 15 July 2015

\section{Introduction}

Calcium $\left(\mathrm{Ca}^{2+}\right)$ channels regulate many essential functions in physiology including gene expression, immune cell function, thrombus formation, muscle contractions, and bone turnover (Fanger et al., 1995; Jackson et al., 2003; Schwarz et al., 2004; Wada et al., 2006; Gwack et al., 2007). The lack of calcium channel activity results in disease states such as immunodeficiency and developmental defects in mineralized tissues (Robinson et al., 2012). Store operated calcium sensors in the endoplasmic reticulum regulate $\mathrm{Ca}^{2+}$ concentrations in the lumen and directly control $\mathrm{Ca}^{2+}$ channels in the plasma membrane (Thompson et al., 2009). Orai1 is an essential subunit of the $\mathrm{Ca}^{2+}$ release-activated

Grant sponsor: American Association of Orthodontists Foundation (AAOF); Grant number: N017774-00.

Drs. Zheng and Zinn contributed equally to this work.

${ }^{*}$ Correspondence to: Petros Papagerakis, Department of Orthodontics and Pediatric Dentistry, Center for Computational Medicine and Bioinformatics, Center for Organogenesis, University of Michigan, Ann Arbor, MI 48109. E-mail: petrosp@umich.edu calcium (CRAC) channel. There is strong evidence that Orail is the pore forming subunit of the CRAC channel and serves as a selectivity filter (Prakriya et al., 2006; Yeromin et al., 2006). When calcium levels in the endoplasmic reticulum are depleted, Orai1 multimerizes and interacts with the stromal interacting molecule (Stim1) to enhance calcium intake into the ER lumen and to activate $\mathrm{Ca}^{2+}$-dependent signal transduction (Thompson et al., 2009). Over-activation of ORAI1 channels leads to large $\mathrm{Ca}^{2+}$ currents into the ER. Two other proteins, ORAI2 and ORAI3, are also present in mammalian cells. However, compared with ORAI1, these two other ORAI proteins produce lower $\mathrm{Ca}^{2+}$ currents when paired with STIM1 (Mercer et al., 2006). It is unclear whether the other ORAI proteins work in conjunction with ORAI1 in a heteromeric channel or they form independent channels in specific cell types (DeHaven et al., 2007).

Article is online at: http://onlinelibrary.wiley.com/doi/10.1002/dvdy. 24307/abstract

(C) 2015 Wiley Periodicals, Inc. 
Based on recent studies, orai 1 has been identified as a primary contributor to the T-cell activation pathway (Feske et al., 2006; McCarl et al., 2009; Thompson et al., 2009). It has also been highlighted in osteoclast differentiation and bone development (Robinson et al., 2012). In fact, mutated Orai1 causes human severe combined immunodeficiency (SCID), a rare disorder that usually results in death before age 1 due to severe infections (McCarl et al., 2009). In these patients, defective orai1 inhibits T and B lymphocyte function. Two surviving patients with an orai 1 mutation show a defined list of symptoms including SCID, ectodermal dysplasia with anhidrosis, global muscular hypotonia, and abnormal enamel formation (McCarl et al., 2009). However, unlike how ectodermal dysplasia normally presents, these patients do not display defects in hair and nail formation. The deciduous and permanent dentitions of these patients have exposed dentin due to use-dependent loss of the enamel layer. The observed defects in enamel fall into the amelogenesis imperfecta (AI) family of diseases and the resulting enamel is soft and hypocalcified. The expression pattern and potential roles of orai 1 in enamel formation remain unclear.

Serving as the protective outer layer for teeth during masticatory forces, enamel is the most mineralized tissue in the body. During tooth formation, the ameloblasts form the enamel layer and surround the entire surface. These cells are eventually lost as the tooth emerges into the oral cavity (Nanci, 2007). The process of ameloblast differentiation and function is multifactorial and involves many players. Ameloblasts go through three main stages of development during enamel formation. The first is the inductive or presecretory stage. During this period, the dentin is not yet mineralized and there is a basal lamina between the inner enamel epithelium and the dental papilla. Odontoblasts then send signals to the inner enamel epithelium (IEE) to induce differentiation of these cuboidal cells into columnar pre-ameloblasts. The second phase of development is the secretory stage. The ameloblasts are now polarized columnar cells that produce enamel proteins. These proteins form the enamel matrix, which is then gradually mineralized with the help of the alkaline phosphatase enzyme from the secretory to the maturation stage. The secretory ameloblasts develop conical processes at the distal end called Tomes' processes, which help to lay down the prismatic layers of enamel. The enamel shape and thickness is determined during the secretory stage of development (Simmer et al., 2010). The final and longest stage of enamel formation is the maturational stage. This final process consists of thickening of the crystals laid down during the formative stage of development and determines the hardness of the enamel layer (Smith, 1998). The ameloblasts are reduced in height, the morphology of their distal ends is altered, and they are still synthesizing small amounts of matrix protein. During the transition from the secretory to the maturation stage, and throughout maturation, approximately $50 \%$ of the ameloblasts are lost due to programmed cell death. The remaining ameloblasts become smooth ended cuboidal cells that serve as a protective layer over the enamel before eruption.

The columnar secretory ameloblasts are responsible for secretion of essential enamel proteins such as enamelin, ameloblastin and amelogenin into the enamel extracellular space, as well as the initial deposition of mineral. In addition, a major function of ameloblasts is $\mathrm{Ca}^{2+}$ transport into the extracellular space where it is incorporated into calcium hydroxyl apatite crystals (Hubbard, 2000). It has been shown through chemical and radiographic studies of rat incisors that a large majority of total calcium is deposited during the maturation stage of ameloblast development (Robinson et al., 1974; Suga, 1982; Kawamoto and Shimizu, 1990; Smith, 1998). However, the mechanism for transport of $\mathrm{Ca}^{2+}$ from the basal and out of the apical membrane of ameloblasts is still undetermined. The first of three theories suggests that the $\mathrm{Ca}^{2+}$ transport occurs paracellularly, through intercellular junctions. The second involves transport of $\mathrm{Ca}^{2+}$ transcellularly through the cytoplasm. The final theory focuses on transport through the ER of ameloblasts (Smith, 1998; Hubbard, 2000). These theories for calcium efflux into the mineralizing enamel remain to be verified.

Our study was initiated to test the hypothesis that Orail controls ameloblast differentiation and function by targeting calcium-dependent gene expression. First, we characterized ORAI1 protein localization in the developing tooth bud of wildtype (WT) mice. Second, we measured the potential effects of Orail shRNA knockdown on cell proliferation and; and evaluate the effects of Orail shRNA knockdown on ameloblast differentiation-related gene expression. This is the first step in understanding how defects in Orail may lead to changes in tooth organogenesis. It will also provide insight on the cause of tooth defects in diseases such as ectodermal dysplasia.

\section{Results}

\section{ORAI1 and ORAI2 Protein Expression in Developing Mouse Teeth, Salivary Glands, Hair Follicle, and Oral Epithelium}

Early embryonic stages of tooth development (E13 and E14) show no ORAI1 or ORAI2 staining in any part of dental tissues. ORAI1 expression becomes apparent in teeth during late embryonic (E17) tooth development (Fig. 1A, B) and continues in newborn stage (NB) (Fig. 1C, D), postnatal day 4 (P4) (Fig. 1E, F) and postnatal day 21 (P21) (Fig. 1G-I). In the embryonic tooth buds (E17), ORAI1 is detected mainly in the internal enamel epithelium but also sporadically in dental papilla and cervical loop area (Fig. 1A, B). In NB mice ORAI1 staining is strong in presecretory ameloblasts but also in stratum intermedium and young odontoblasts (Fig. 1C, D). In P21 teeth, ORAI1 is detected in secretory and maturation ameloblasts (Fig. 1G-I), and differentiated odontoblasts (Fig. 1H). High magnification pictures show clear cytoplasmic/membranous staining within the limits of light microscopy. No significant signal is found when rabbit serum is used instead of primary antibody in mouse teeth serial sections (negative control) at any age as shown here for P21 (Fig. 1J). In postnatal craniofacial tissues of P21 mice ORAI1 is also detected in salivary glands (Fig. 1K), hair follicles (Fig. 1L) and oral epithelial cells (Fig. 1F).

ORAI2 staining is first detected at E17 mainly expressed in the IEE area and pre-ameloblastic cells (Fig. 2A, B). In teeth of newborn (NB) mice ORAI2 is expressed strongly in differentiated ameloblasts (Fig. 2C) and oral epithelial cells (Fig. 2D).

\section{Orai1 Knockdown on Ameloblastic HAT-7 by shRNA}

Orail knockdown (KD) in HAT-7 ameloblastic cells using the shRNA lentiviral particles decreased Orail mRNA expression by 80\% (Fig. 3A). Western blot analysis (Fig. 3B) followed by densitometry measurement (Fig. 3C) using ImageJ software (NIH), shows significant knockdown of ORAI1 at the protein level. Scrambled shRNA is used as a control. We also examined by immunofluorescence (IF) staining the levels of ORAI1 protein in 

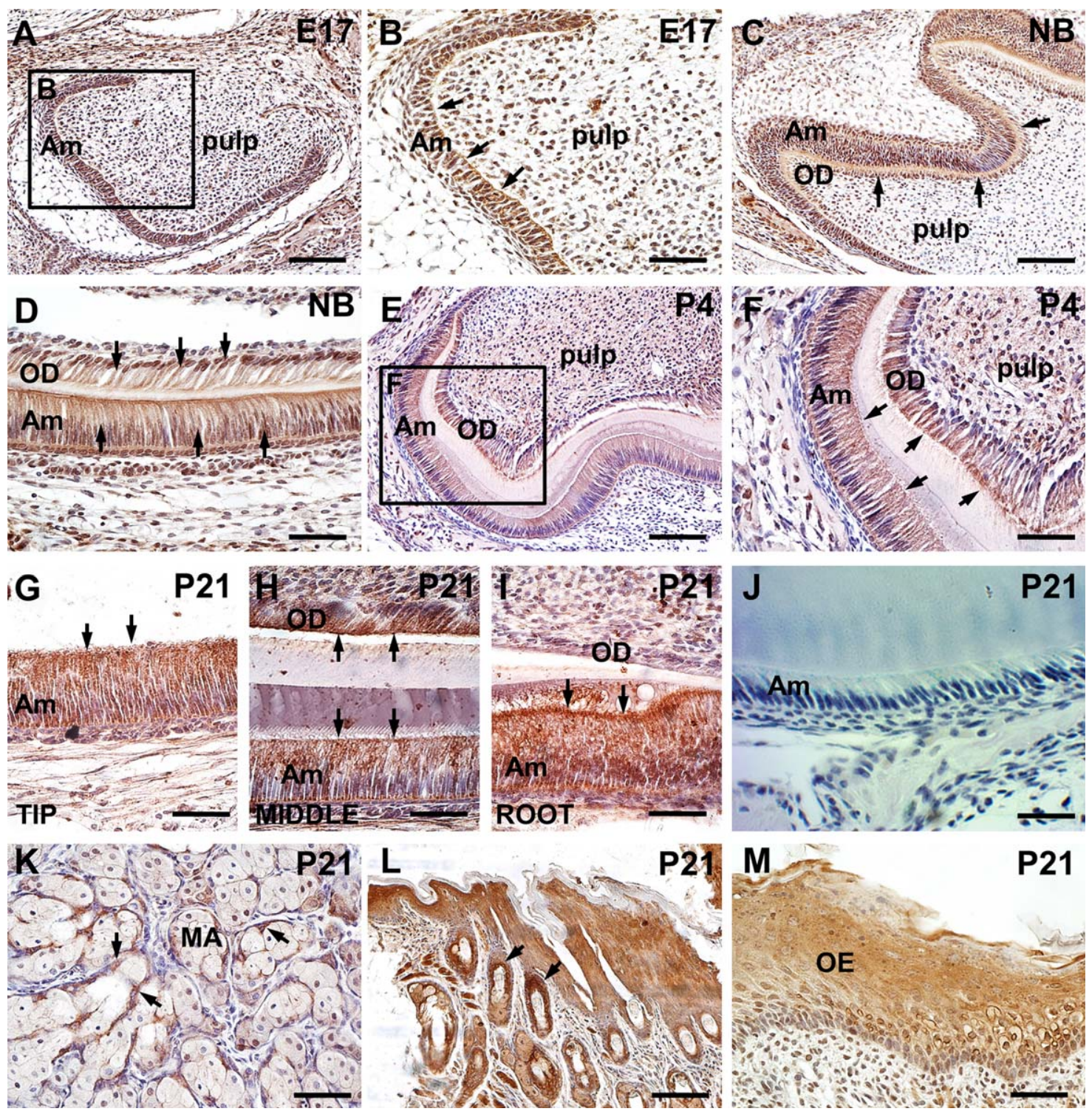

Fig. 1. ORAl1 expression in the developing orofacial tissues. Immunohistochemistry (IHC) staining for ORAl1 was performed on tooth sections at embryonic day E17 (A, B), newborn NB (C, D), postnatal P4 (E, F), and postnatal P21 (G-M). In E17, ORAl1 is mainly expressed in preameloblastic cells (A, B). In NB (C, D), P4 (E, F), and P21 (G-J), both ameloblasts and odontoblasts show positive staining of ORAl1 in molar (C-F, $\mathrm{J})$ and in the incisor root $(\mathrm{G})$, middle $(\mathrm{H})$, and tip (I) part. Replacement of the first antibody with rabbit serum results in lack of staining $(\mathrm{J})$ serving as negative control. ORAl1 also is detected on the cell membrane mucous acini in the salivary glands (K), skin epidermis (L), hair follicle (L, arrow) and stratified squamous nonkeratinized epithelium (M). Arrows indicate the positive signal of IHC. Am, ameloblast; OD, odontoblast; MA, mucous acini; Scale bars $=100 \mu \mathrm{m}$ in A, E, K, L, M; $50 \mu \mathrm{m}$ in B-D, F-J.

scrambled shRNA transduced cells (control) and Orail shRNA transduced HAT-7 cells. The IF data further confirm the specificity of the ORAI1 antibody showing a clear down-regulation of ORAI1 protein immunofluorescence staining in Orai1 shRNA transduced cells compared with the control (Fig. 3D).

\section{Effect of Orai1 Knockdown on HAT-7 Ameloblast Cell Proliferation Rate}

The proliferation of Orail shRNA knockdown cells compared with control cells is assessed by MTT assay and cell count. The MTT assay shows a significant decrease in proliferation rate between the two groups (control cells cultured in normal medium and orai1 shRNA knockdown cells). The greatest rate difference occurs between days 2 and 4. Proliferation converged at day 7 when cell confluence reached 100\%. Similar effects are observed when using HAT-7 cells transduced with nonspecific shRNA lentivirus (scramble shRNA) and compared with Orai1 shRNA transduced cells. Cell counting demonstrates a significant decrease in proliferation rate in the Orail shRNA transduced cells compared with the control cells transduced with nonspecific shRNA lentivirus (Fig. 3E).

\section{Effect of Orai1 Knockdown on Ameloblast Gene Expression}

The effects of Orail in ameloblasts are examined in two different time points after virus transduction and selection. We collected 


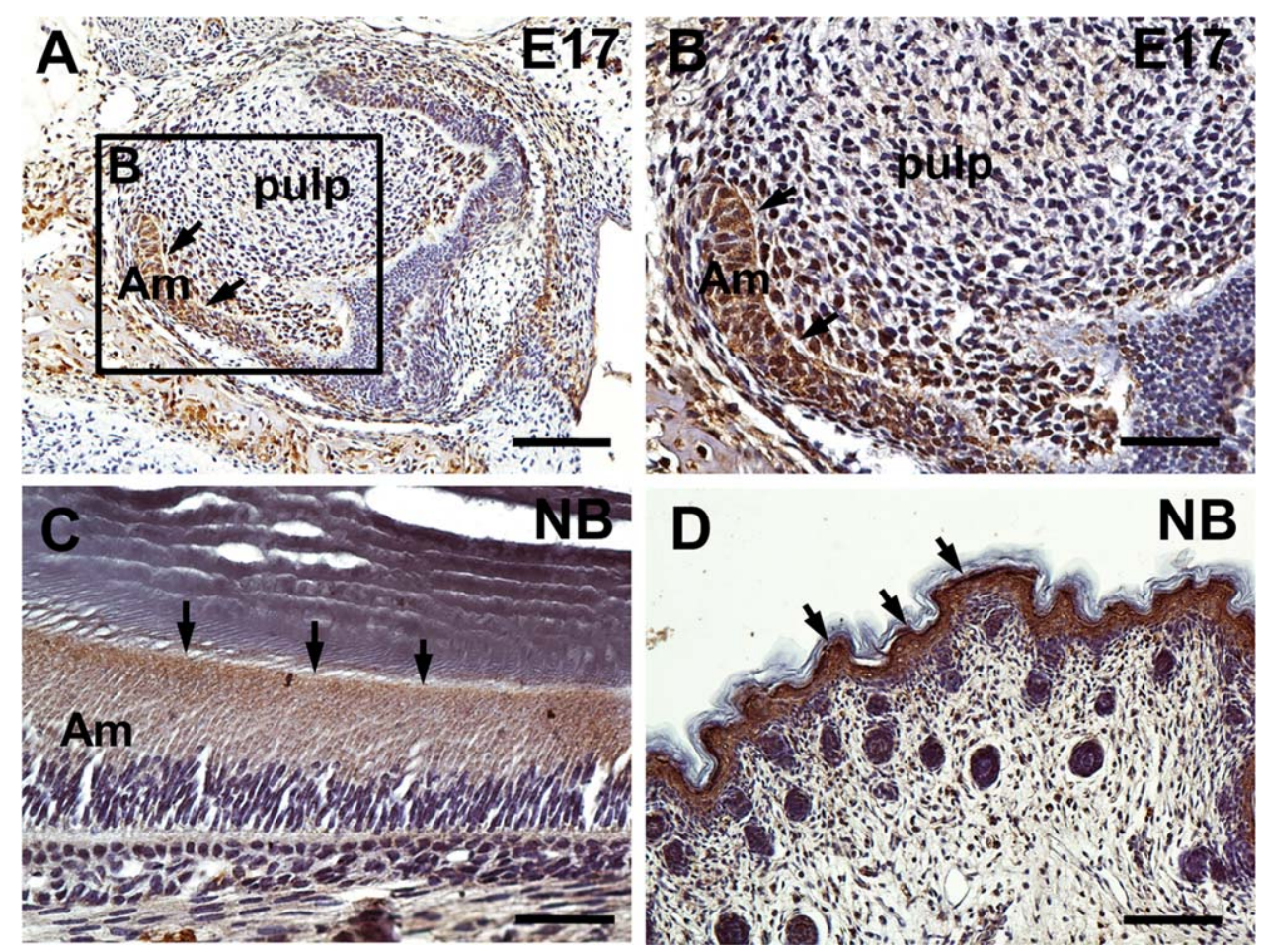

Fig. 2. ORAl2 expression in the developing orofacial tissues. Immunohistochemistry staining shows expression of ORAl2 in cervical loop and pre-ameloblastic cells of molar (A, B) and ameloblastic cells of incisor (C) teeth at E17 (A, B) and NB (C). ORAl2 expression is also detected in skin epidermis but not in hair follicle (D). Am, ameloblast; Scale bars $=100 \mu \mathrm{m}$ in A, D; $50 \mu \mathrm{m}$ in B, C.

RNA from postconfluent cells 4 weeks after the end of selection (Fig. $4 \mathrm{~A}-\mathrm{H}$ ) and 2 weeks after the end of selection. Both data show consistent trends of gene expression regulation. Orail down-regulation led to significant decreased expression of an early ameloblast differentiation marker, i.e., dentin sialoprotein (DSPP) (Fig. 4A) and two enamel matrix proteins, i.e., amelogenin (Fig. 4B) and ameloblastin (Fig. 4C), both involved in enamel secretion and control of the enamel thickness. Orail downregulation results also in down regulation of metallo-proteinase 20 (MMP20) RNA (Fig. 4D), another marker of secretory ameloblasts.

Ameloblast differentiation and enamel maturation are directly regulated by $\mathrm{pH}$ and ion transport (Lacruz et al., 2013). Among the genes involved in ion transport in ameloblasts, cystic fibrosis transmembrane conductance regulator (Cftr) and carbonic anhydrase 2 (Car2) are significantly up-regulated at the onset of the maturation stage of amelogenesis when compared with the secretory stage and are considered definitive markers of maturation stage ameloblasts (Lacruz et al., 2013). To evaluate the potential effects of Orail in maturation stage ameloblasts, we measured the relative expression levels of calcium related genes, Car2, plasma membrane calcium (Ca2+) ATPase 1 (Pmca1) and Cftr. Car2 is widely expressed in ameloblasts regulating the reversible hydration of carbon dioxide into bicarbonate and protons ( $\mathrm{CO} 2+$ $\mathrm{H} 2 \mathrm{O} \Longleftrightarrow \mathrm{HCO}-+\mathrm{H}+$ ). Pmca 1 is a transport protein that functions to remove $\mathrm{Ca} 2+$ from the cell and is a known target of Orail in other systems (Baryshnikov et al., 2009). Cftr is a protein involved in the transport of chloride ions across cell membranes and mice with Cftr mutation show a lack of calcium influx during enamel maturation resulting in enamel hypomineralization (Sui et al., 2003).
Both, Car2 and Pmca1 are found down-regulated in HAT-7 Orail knockdown cells (Fig. 4E, G) but only Pmca 1 shows a statistically significant down regulation (Fig. 4E). In contrast, Cftr expression is up-regulated in HAT-7 orai1 knockdown cells (Fig. 4F).

To evaluate potential effects of Orai1 in ameloblast maturation and mineralization, we also evaluated alkaline phosphatase (ALP) levels, a marker of maturation ameloblasts, by reverse transcriptase-polymerase chain reaction (RT-PCR) (Fig. 4H) and by in situ staining (Fig. 4I). ALP RNA levels are found slightly increased in Orail knockdown cells compared with control cells but this difference is not statistically significant (Fig. $4 \mathrm{H}$ ). The alkaline phosphatase in situ staining is performed at 4 weeks of cell culture. Compared with the control group without differentiation media and the control group with differentiation media, the Orail KD cells cultured with differentiation media show a slightly stronger ALP staining. However, the difference between the control and KD plates is not significant (Fig. 4I).

\section{Discussion}

Orail is a transmembrane protein found in the plasma membrane of many mammalian tissues and cell types. It is a four-domain subunit that is part of the $\mathrm{Ca} 2+$ release activated Ca2 + (CRAC) channels (Vig et al., 2006; Zhang et al., 2006; Putney, 2007). Orail was first reported by Feske et al. through genome mapping in a family with a form of immunodeficiency due to malfunction of CRAC (Feske et al., 2006). Calcium depletion in the ER leads to aggregation of the stim 1 protein with orai 1 and allows calcium entry (Thompson et al., 2009). Recent studies have shown ORAI1deficient patients present with a form of anhidrotic ectodermal 


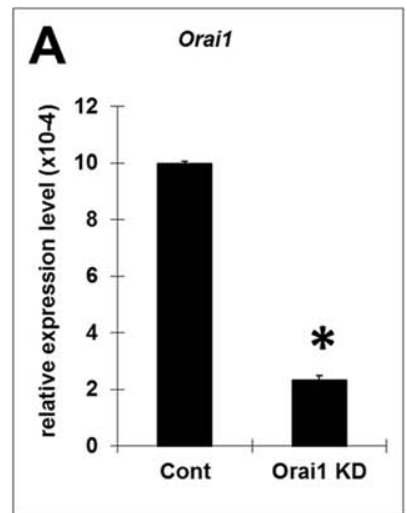

B

Mock KD

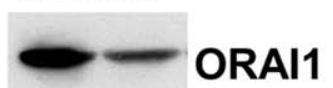

D
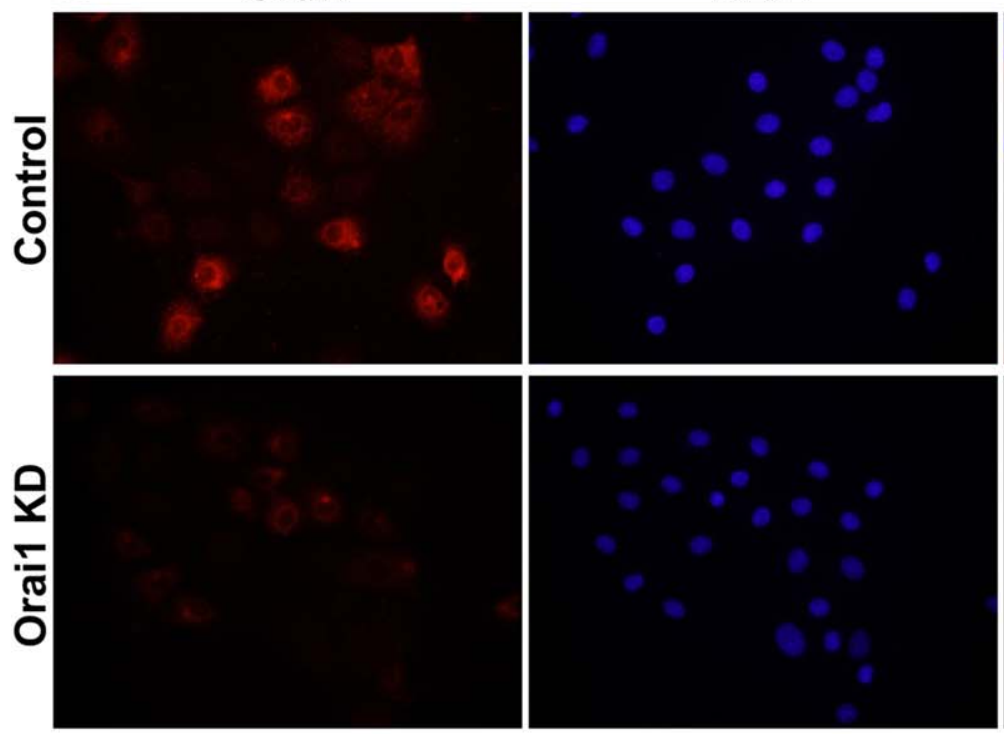

Growth Speed

E

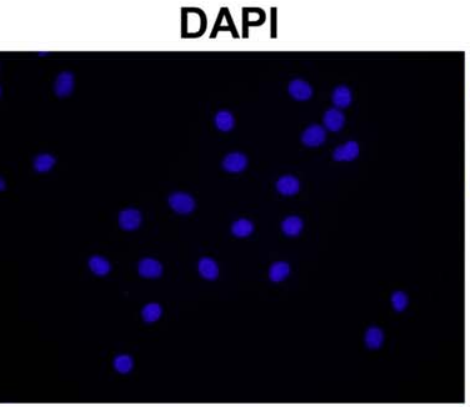

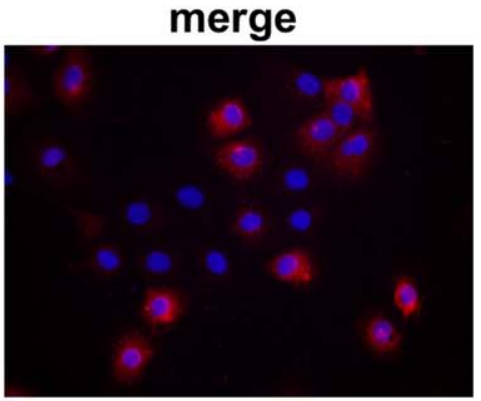
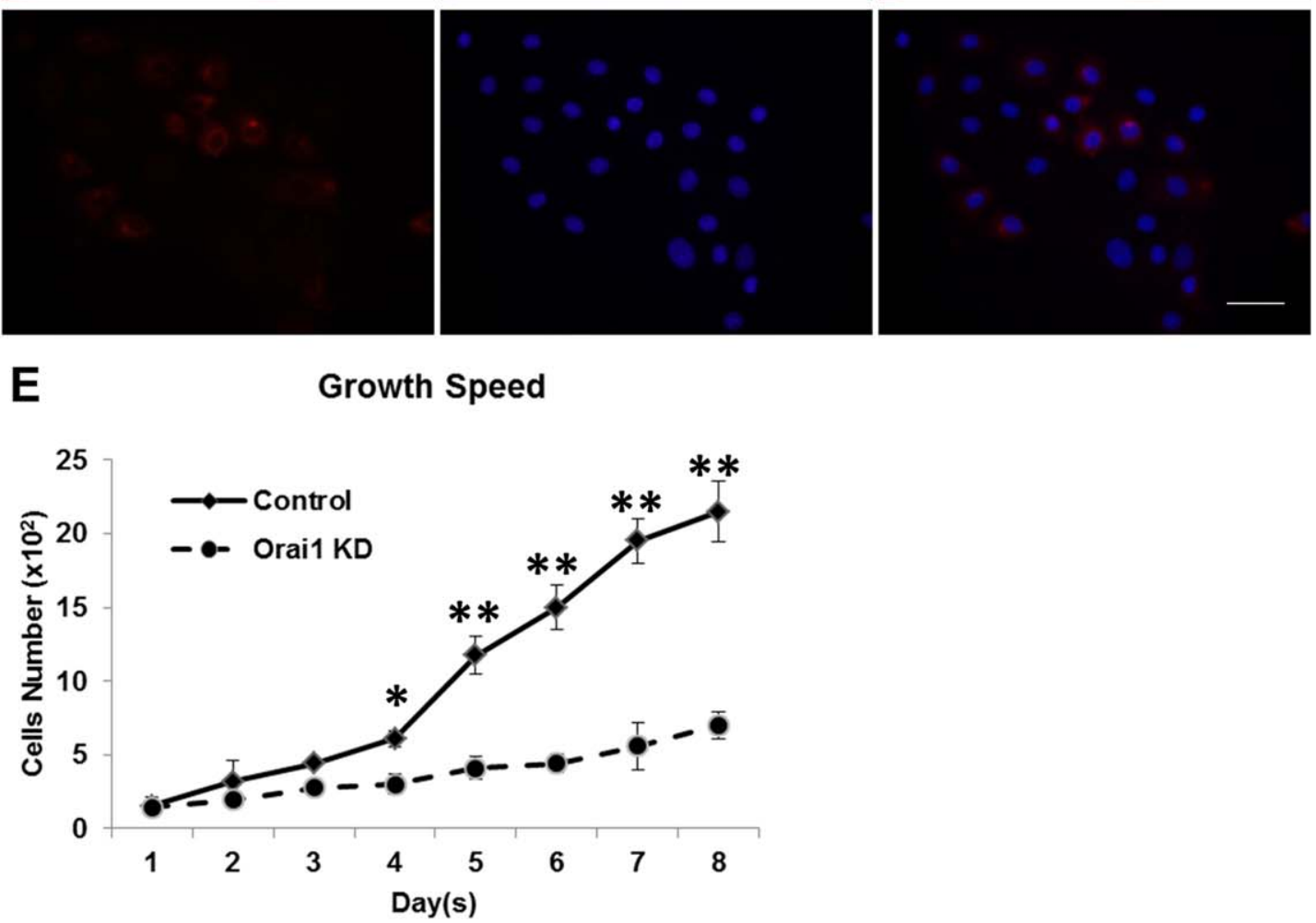

Fig. 3. Comparison between relative expression levels of Orai1 mRNA (A) and protein (B) expression levels in control cells (scramble shRNA, "Mock") and Orai1 knockdown cells. Densitometry analysis using the NIH ImageJ software (C), performed on the Western blot (B), shows a significant decrease in ORAl1 protein expression in the knockdown cells. The representative data are presented as means \pm SD from at least three independent replicates where data from cells transfected with Orai1 shRNA were compared with cells transfected with control virus (scramble shRNA). Error bars indicate \pm standard error. A Student's $t$-test was used to determine statistical significance $\left({ }^{\star *} P<0.01\right)$. A clear down-regulation of ORAl1 protein immunofluorescence staining is found in Orai1 shRNA transduced cells (ORAl1 KD) when compared with the control cells (D). HAT-7 cell growth speed measured by cell counting demonstrates a significant proliferation decrease in the Orai1 shRNA transduced cells (Orai1 KD) when compared with cells transduced with nonspecific shRNA lentivirus (control) (E). 

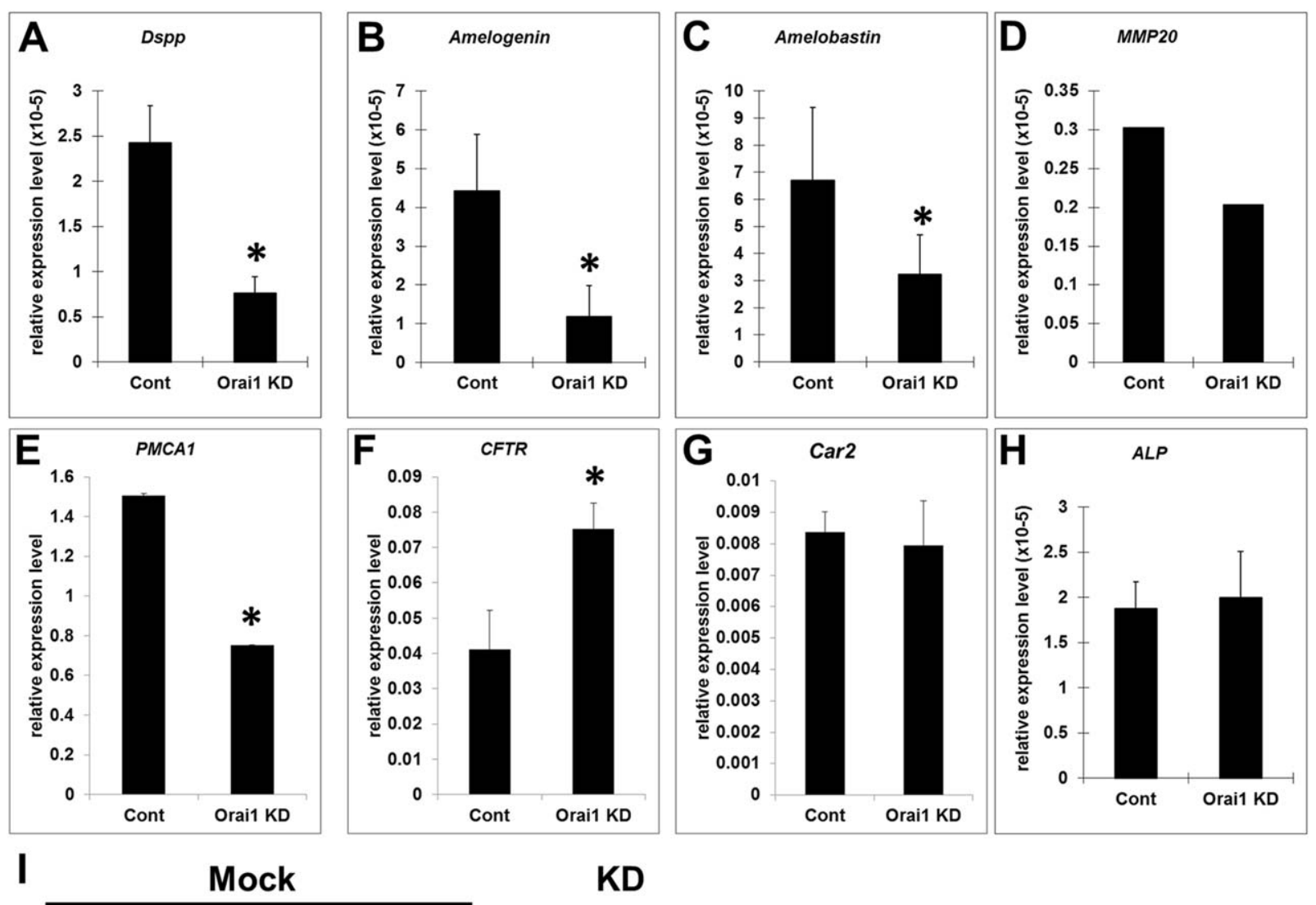

\section{KD}

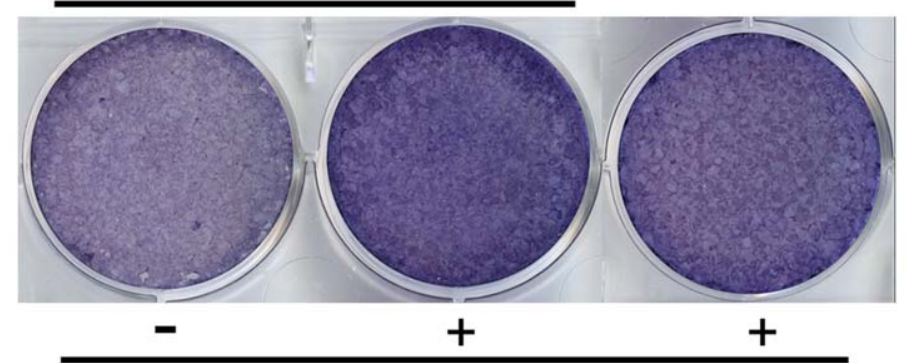

\section{B-glycerophosphate + ascorbic acid (4wks)}

Fig. 4. Orai1 regulates gene expression during ameloblast differentiation. Orai1 knocked-down (KD) by Orai1 shRNA transduction results in changes in RNA levels of key ameloblast differentiation genes at postconfluent cells growing in mineralization medium for 4 weeks after selection. In Orai1 KO cells Dspp (A), Amelogenin (B), Ameloblastin (C), metalloproteinase 20 (MMP20) (D), and plasma membrane calcium (Ca2+) ATPase 1 (Pmca1) (E) showed a statistically significant down-regulation at the RNA levels. In contrast, cystic fibrosis transmembrane conductance regulator (Cftr) (F) RNA levels were found up-regulated in Orai1 KO cells. Carbonic anhydrase 2 (Car2) (G) and alkaline phosphatase (A/p) (H) levels were not significantly affected in Orai1 KD cells. Error bars indicate \pm standard error. A Student's $t$-test was used to determine statistical significance $\left({ }^{*} P<0.05\right)$. ALP in situ staining in HAT-7 cell shows no significant changes in color density in knockdown and control groups after culture with differentiation medium (I).

dysplasia, which includes characteristics of amelogenesis imperfect (McCarl et al., 2009). These patients also display severe immune deficiency, myopathy and anhydrosis. Orai1-/- knockout mice were also generated and consistently displayed immunodeficiency, overall smaller sizes and diminished enamel layer in their teeth (Gwack et al., 2008, Robinson et al., 2012). Although Orail is found to have almost ubiquitous expression in many cell types, the symptoms of Orail deficiency involve specific mostly ectodermal derived organs. Much attention has been devoted to finding the role of orai1in the development and function of these specific tissues. This study is aimed at exploring the essential role of Orail in ameloblast differentiation and function.
Previous studies have shown that Orai1 is an important pore subunit of the CRAC channels and may play a role in controlling gene expression in cell differentiation (Zhou et al., 2011; Robinson et al., 2012). Our data show that ORAI1 protein (and OARI2 in less extend) is differentially expressed during odontogenesis and strongly expressed mainly by postnatal ameloblasts and odontoblasts suggesting a key role on the enamel and dentin formation.

Despite this increase in interest in the CRAC system and Orail, it is still unclear what the downstream effectors are of the Orail gene expression. Although our data suggest that orai1 altered expression can cause amelogenesis imperfecta by dysregulating 
ameloblast gene expression, we do not know the exact genetic pathways involved. In our system of Orail deletion, one possibility is that the NFAT pathway is dysregulated. NFAT pathway is important in its regulation by the calcium/calmodulin-dependent serine phosphatase calcineurin complex (Hogan et al., 2003). Although this pathway has mainly been documented in association with the immune response, this could be one of the missing links in the control of gene expression by orai1 dependent calcium entry in ameloblasts. NFAT is able to activate a large number of genes during the immune response as a result of a rise in intracellular calcium levels. There is evidence that the NFAT pathway is very versatile and is active in cell types other than immune cells (Graef et al., 2003, Hill-Eubanks et al., 2003). Although these studies were performed in muscle and nerve cells, more work needs to be done in ameloblasts to show whether the downstream effector of the Orail/CRAC complex is indeed connected to the NFAT pathway. NFAT pathway may be a component of the proliferarion decrease observed in Orail shRNA down-regulated HAT-7 cells. Of interest, despite the fact that the knockdown only results in partial elimination of orai1 protein from the HAT-7 cell line, we were able to see dramatic differences in proliferation rate of ameloblasts. It is actually seems that orai 1 is crucial in controlling the successful proliferation of HAT-7 cells. We used two techniques to quantitate the difference between the proliferation of normal control HAT-7 cells and that of the orai1 knockdown cell line. Using both the MTT assay and a cell count comparison, we were able to see a significantly lower proliferation rate in the Orail knockdown cells. Further studies are needed to clarify the mechanisms by which Orail controls cell proliferation.

We also show that Orail plays a potential key role in ameloblast differentiation and enamel maturation. The expression of Orail during the ameloblast development may indicate a connection between calcium influx, gene expression and ameloblast differentiation. By regulating ameloblast gene expression, such as amelogenin and ameloblastin (this study), Orail may contribute to the control of enamel thickness. Furthermore, by controlling the expression of genes encoding calcium-signaling molecules such as Pmca1 and in a less extend Car2 (this study), Orail goes beyond the CRAC pathway to regulate calcium homeostasis and enamel mineralization. Car2 and Pmca1 are both important in the efflux of calcium out of the cell and into the enamel matrix, (Zhang et al., 2006; Lacruz et al., 2013). Car2 is also important for $\mathrm{pH}$ regulation. $\mathrm{HCO}$ - neutralizes $\mathrm{H}+$ released during enamel crystal formation, permitting continued crystal growth. Our data also suggest that Orai1 may influence enamel maturation by regulating other ion channel expression such as CFTR (Bronckers et al., 2010) and also by regulating the expression of enamel proteinases such as Mmp20. Of interest, a recent study focused on solute carrier family (Hill-Eubanks et al., 2003), member (Bergendal, 2010) (SLC24A4) and ORAI1 (Wang et al., 2014). SLC24A4 is a $\mathrm{Na}^{+} / \mathrm{K}^{+} / \mathrm{Ca}^{2+}$ transporter that exchanges intracellular $\mathrm{Ca}^{2+}$ and $\mathrm{K}^{+}$for extracellular $\mathrm{Na}^{+}$. This study suggests that mutations in SLC24A4 cause enamel malformations similar to those found in ORAI1 deficient patients. They hypothesized that SLC24A4 is the missing link that allows $\mathrm{Ca}^{2+}$ efflux out of the cell and into the mineralizing enamel matrix. They also hypothesized that the efflux of calcium through this channel lowers intracellular and $\mathrm{ER} \mathrm{Ca}^{2+}$ stores and activates the influx of $\mathrm{Ca}^{2+}$ through the proximal membranes by means of the ORAI1/CRAC system. Taken together, these findings and ours suggest that orai1 may play a key role during both the secretory and the maturation stages of enamel formation. Thus, calcium homeostasis may control the expression of important enamel forming proteins and also regulate the amount of calcium secreted at the enamel matrix. This potential role of Orail could explain the finding of amelogenesis imperfecta in patients that have an ORAI1 deficiency.

The mechanism behind ameloblast differentiation and function is still poorly understood. This study aims to contribute in uncovering a critical role for the newly discovered protein orai1 in the development, differentiation and function of ameloblasts. Immuno-staining of embryonic mouse tooth sections (E13-E14) showed that ORAI1 is not expressed in early embryonic dental tissues but its expressions is initiated in later embryonic stage E17 in inner enamel epithelium cells, the precursors of ameloblasts. The localization of orai 1 in ameloblasts and odontoblasts is first observed in the beginning of differentiation stages at newborn (NB) mice. ORAI1 protein expression continues on enameland dentin-forming cells throughout tooth development. Our results suggest that ORAI1 is mainly active during the secretory and maturational stages of ameloblast development and throughout odontoblast differentiation. It is during the secretory and maturational stages that ameloblasts produce the majority of the enamel matrix proteins. Extracellular proteins form the enamel matrix, which is then gradually mineralized from the secretory to the maturation stage with the help of alkaline phosphatase enzyme as well as by a complex network of ion channels. The enamel shape and thickness is determined during the secretory stage of development (Simmer et al., 2010). During the transition from the secretory to the maturation stage, and throughout maturation, approximately 50\% of the ameloblasts are lost due to programmed cell death. The final maturational process consists of thickening of the crystals laid down during the formative stage of development and determines the hardness of the enamel layer (Smith, 1998). It is clear that the expression of orai1 correlates with the most important stages of ameloblast function and enamel development. The precise roles of CRAC channels in tooth organogenesis remain to be discovered. Future studies are still required to determine the exact downstream effectors of orai1 in controlling gene expression. Nevertheless, our results show for the first time that Orail influences the expression of ameloblast differentiation markers and should be considered an important regulator of gene expression and matrix formation in enamel formation.

\section{Experimental Procedures}

\section{Animals}

Animal use was approved by the Animal Welfare Committee of the University of Michigan. Mouse heads from WT C57BL/6 mice between E13 (cap stage of tooth development) and P21 were fixed with formaldehyde, demineralized, embedded in paraffin, and then sectioned. For immunohistochemistry, serial sections (7 $\mu \mathrm{m}$ thick) of E13, E14, E17, NB, P4, and P21 mouse heads were used.

\section{Immunohistochemistry}

Mouse heads were sectioned at the incisor and molar tooth buds and immune-stained for orai1 and orai2 protein localization using rabbit anti-ORAI1 (1:250, ab59330, Abcam, Cambridge, 


\begin{tabular}{|c|c|c|c|c|}
\hline Gene name & & $5^{\prime}$ - Sequence $-3^{\prime}$ & Product size (bp) & GenBank no. \\
\hline \multirow[t]{2}{*}{$\beta$-actin } & Forward & AAGTACCCCATTGAACACGG & 257 & NM_031144 \\
\hline & Reverse & ATCACAATGCCAGTGGTACG & & \\
\hline \multirow[t]{2}{*}{ Orai1 } & Forward & GCTCTGCTGGGTGAAGTTCT & 198 & NM_001013982 \\
\hline & Reverse & AAGTGAACGGCAAAGACGAT & & \\
\hline \multirow[t]{2}{*}{ Amelogenin } & Forward & TACCACCTCATCCTGGGAGC & 164 & U01245 \\
\hline & Reverse & CTGTTGAGACAGCACAGGGA & & \\
\hline \multirow[t]{2}{*}{ Ameloblastin } & Forward & CAGAAGGCTCTCCACTGCAA & 248 & NM_012900 \\
\hline & Reverse & CCCCAAGGGTGTGGTAACAT & & \\
\hline \multirow[t]{2}{*}{$D S P P$} & Forward & GTGCTAAAGAACGCGAGGAC & 200 & NM_012790 \\
\hline & Reverse & GTCTCCATTCTGGCTCGTGT & & \\
\hline \multirow[t]{2}{*}{ MMP20 } & Forward & CGACAATGCTGAGAAGTGGA & 202 & NM_001106800 \\
\hline & Reverse & GGGTCCATATAACGCTTGGA & & \\
\hline \multirow[t]{2}{*}{$P M C A 1$} & Forward & GGCGACTTTGGCATCACACT & 120 & NM_053311 \\
\hline & Reverse & TTTCAACTTGGTGCAAATTCCA & & \\
\hline \multirow[t]{2}{*}{ CFTR } & Forward & TGTGGATGGGGGTTATGTGC & 180 & NM_031506 \\
\hline & Reverse & AGGACTACTGTGCAACCAGC & & \\
\hline \multirow[t]{2}{*}{ Car2 } & Forward & AAGAGCAACGGACCAGAGAA & 206 & NM_019291 \\
\hline & Reverse & GCAAAGTCCTGGGAGTCATC & & \\
\hline \multirow{2}{*}{$A L P$} & Forward & TGATCACTCCCACGTTTTCA & 202 & NM_013059 \\
\hline & Reverse & CTGGGCCTGGTAGTTGTTGT & & \\
\hline
\end{tabular}

MA) or rabbit anti-Orai2 (1:500, PA5-20369, Thermo Scientific, Rockford, IL).

Primary antibodies were diluted with phosphate buffered saline with $1 \%$ bovine serum albumin. Following washes with phosphate buffered saline (PBS) $+1 \%$ bovine serum albumin (BSA), the sections were incubated for $30 \mathrm{~min}$ with mouse anti-rabbit IgG biotinylated secondary antibody. Secondary antibody was followed by washes with PBS $+1 \%$ BSA, then incubation with an avidin-biotin-peroxidase complex. Visualization with 33 'diaminobenzidine (DAB) was performed based on the peroxidase substrate kit DAB (SK-4100, Vector Laboratories, Burlingame, CA) instructions, and the reaction was stopped before detection of nonspecific staining in control preimmune serumtreated sections. The histological staining was completed with a hematoxylin counterstain. Sections were then mounted and photographed on an Olympus microscope. Serial sections where rabbit serum was used instead of primary antibody on E17- and P21-old mouse teeth was used a control.

\section{Cells}

Sub-confluent cells were thawed and cultured in high glucose DMEM supplemented with 100 units/ml penicillin G, $100 \mu \mathrm{g} / \mathrm{ml}$ streptomycin (Invitrogen, Carlsbad, CA) and 10\% (v/v) fetal bovine serum (SAFC Biosciences, Lenexa, KS). Two sets of ameloblastic HAT-7 cells were grown in differentiation media for 10 days in the presence of pLK0.1-puro empty lentivirus (scramble shRNA) and Orai1 shRNA lentiviral particles (viruses were purchased as a kit through Sigma [\#SHCLNV-NM_175423, MISSION shRNA Lentiviral Transduction Particles]). One set of cells was assayed for proliferation using an MTT assay. Another set of cells was placed on 96-well plate (100 cells per well) and cultured in DMEM/F12 medium for 8 days. Cell numbers were counted on every day. Total RNA was collected from another set of cells cultured in mineralization medium at two post confluent time points ( 2 and 4 weeks after the end of the selection) and assayed for
mRNA expression of ameloblast differentiation markers using quantitative real-time PCR (qRT-PCR).

\section{Transduction}

For transduction studies, HAT-7 cells were plated at 70\% confluence in 6-well plates. After changing to serum-free DMEM/F-12 without antibiotics overnight, the control Lentivirus and Orail shRNA knockdown viruses were transducted into control and knockdown HAT-7 cells, respectively, following Sigma Aldrich Transduction protocol. A total of $8 \mu \mathrm{g} / \mathrm{ml}$ of hexadimethrine bromide was added to improve the transduction efficiency. The Lentivirus particles were added using the desired multiplicity of infection (MOI), which is the desired number of transducing particles per cell. The cells were then incubated for $24 \mathrm{hr}$ before selection was performed. After incubation, puromycin at a concentration of $1 \mu \mathrm{g} / \mathrm{ml}$ was used for selection of transduced cells. The puromycin-containing medium was replaced every 3 days until the resistant colonies were recognized.

\section{qRT-PCR and RNA Expression}

RNA was extracted from the HAT-7 cells using the QIAGEN RNeasy system (QIAGEN; Izasa SA, Barcelona, Spain). Two micrograms of total RNA from the control and knockdown samples were reverse transcribed with TaqMan reverse transcription reagents (Applied Biosystems, Branchbury, NJ), following the manufacturer's recommendations. The resulting cDNA was then amplified by RT-PCR or real time quantitative RT-PCR (qRT-PCR) using AmpliTaq Gold DNA Polymerase (Applied Biosystems). The RT-PCR products were subcloned into pGEM-T Easy vector (Promega, Madison, WI) and confirmed by sequencing. For RNA quantification, qRT-PCR amplifications were performed at $95^{\circ} \mathrm{C}$ for $30 \mathrm{sec}, 60^{\circ} \mathrm{C}$ for $30 \mathrm{sec}$, and $72^{\circ} \mathrm{C}$ for $30 \mathrm{sec}$ using specific primers for the ameloblast specific genes. The PCR primers are based on the published cDNA sequences shown in Table 1 . The relative 
expression levels for each gene were calculated based on the expression levels of beta-actin and the differences were presented in graphs using the $2-$ DDCT method. $P$-values were calculated using a two-sample $t$-test.

\section{Proliferation Assays}

An MTT assay was performed to quantify the differences in proliferation rate between the cell lines. The MTT (4,5-dimethylthiazol-2-yl)-2,5-diphenyltetrazolium bromide) assay is a colorimetric assay that quantifies cell viability. The reaction depends on tetrazolium dye reduction by $\mathrm{NAD}(\mathrm{P}) \mathrm{H}$-dependent oxidoreductase enzymes, which are active in the cytosolic compartment of the cell. One hundred microliters of $0.45 \mathrm{~g} / \mathrm{L}$ MTT solution was added to wells. Cells were incubated at $37^{\circ} \mathrm{C}$ for $45-$ $60 \mathrm{~min}$ to allow color development and thereafter, $100 \mu \mathrm{l}$ of $20 \%$ sodium dodecyl sulfate (SDS) in dimethyl formamide $\mathrm{H}_{2} \mathrm{O} \quad 1: 1$ solution was added to the wells. Plates were incubated overnight at $37^{\circ} \mathrm{C}$ to solubilize the formazan products. Absorbances were measured at the wavelength of $570 \mathrm{~nm}$. The levels of blue color development in the control wells were designated as 100\% viability, and all further comparisons were based on that reference level. Blank values, indicating the absorbance of MTT and 20\% SDS in DMF:H20 1:1 solutions only, were subtracted from all samples.

\section{ALP Staining}

For alkaline phosphatase (ALP) staining, transducted cells were cultured in 6-well plates. The cells were incubated in DMEM with or without AA and GP for 4 days. Thereafter, cells were fixed with 10\% formalin for $15 \mathrm{~min}$, washed with PBS for three times, and stained with NBT-BCIP (B5655, Sigma) for $30 \mathrm{~min}$ at 37 degree.

\section{Western Blot Analysis}

Cells are washed twice with PBS before being lysed for 20 min on ice in RIPA lysis buffer. Total proteins $(50 \mu \mathrm{g})$ per lane are separated by SDS-polyacrylamide gel electrophoresis, and transferred to a polyvinylidene difluoride membrane (Millipore, Billerica, MA). The membrane is blocked with 5\% nonfat milk, incubated overnight at $4^{\circ} \mathrm{C}$ with the primary antibody against ORAI1 (1:500). Anti-glyceraldehyde-3-phosphate dehydrogenase (GAPDH) antibody $(1: 2,500$, Abcam, ab9485) is used to determine the loading control. The membranes are incubated for $1 \mathrm{hr}$ at room temperature with a horseradish peroxidase-conjugated anti-mouse or antirabbit antibody, after washing. Bound antibodies are visualized by enhanced chemiluminescence detection system. All bands are measured by densitometry and normalized to GAPDH (mean$\mathrm{s} \pm$ standard error of three or four experiments) using the Image software.

\section{Immunocytofluorescence}

HAT-7 cells are cultured on glass slides before fixation. After blocking of no-specific antigens using 3\% bovine serum albumin (BSA), cells are incubated with rabbit anti-ORAI1 (1:250, ab59330, Abcam, Cambridge, MA) for $1 \mathrm{hr}$ at room temperature. Next, a fluorescent Alexa Fluor 594 goat anti-rabbit (Invitrogen) secondary antibody is applied to all sections followed by DAPI (4',6-diamidine-2-phenylidole-dihydrochloride) counter staining.
Sections are then mounted with Prolong Gold antifade reagent (Invitrogen). All sections are examined and then photographed on an Olympus microscope. As negative controls, both omission of primary antibody and omission of secondary antibodies are used. Normal rabbit IgG instead of the primary antibody is also used for determination of nonspecific binding of antibodies.

\section{Statistics}

We used a Student's unpaired $t$-test for the statistical analysis of our RT-PCR, MTT assay, and cell count data. Each experiment was performed at least twice and the representative data are presented as means \pm SD from at least three independent replicates.

\section{Acknowledgments}

This project is an active collaboration between Dr. Papagerakis (University of Michigan) and Dr. Nervina. The participation of Dr. Lefkelidou is supported by educational and research collaborations between the Department of Orthodontics and Pediatric Dentistry at the University of Michigan (Dr. Papagerakis) and the Department of Pediatric Dentistry at the Aristotle University of Greece (Chair: Dr. Nikolaos Kotsanos). We have also received the following grant support: American Association of Orthodontists Foundation (AAOF) awarded to P.P./J.N.; Delta Dental Foundation training grant to V.Z./P.P.; University of Michigan Rackham Graduate School Fellowship to V.Z.; Islamic Bank Foundation fellowship to T.B.; and University of Michigan UROP training funds to N.T./S.P./ P.P.

\section{References}

Baryshnikov SG, Pulina MV, Zulina A, Linde CI, Golovina VA. 2009. Orai1, a critical component of store-operated $\mathrm{Ca} 2+$ entry, is functionally associated with $\mathrm{Na}+/ \mathrm{Ca} 2+$ exchanger and plasma membrane $\mathrm{Ca} 2+$ pump in proliferating human arterial myocytes. Am J Physiol Cell Physiol 297:C1103-C1112.

Bergendal B. 2010. Oligodontia ectodermal dysplasia-on signs, symptoms, genetics, and outcomes of dental treatment. Swed Dent J Suppl 13-78:7-8.

Bronckers A, Kalogeraki L, Jorna HJ, Wilke M, Bervoets TJ, Lyaruu DM, Zandieh-Doulabi B, Denbesten P, De Jonge H. 2010. The cystic fibrosis transmembrane conductance regulator (CFTR) is expressed in maturation stage ameloblasts, odontoblasts and bone cells. Bone 46:1188-1196.

Dehaven WI, Smyth JT, Boyles RR, Putney JW. 2007. Calcium inhibition and calcium potentiation of Orai1, Orai2, and Orai3 calcium release-activated calcium channels. J Biol Chem 282: 17548-17556.

Fanger CM, Hoth M, Crabtree GR, Lewis RS. 1995. Characterization of $\mathrm{T}$ cell mutants with defects in capacitative calcium entry: genetic evidence for the physiological roles of CRAC channels. J Cell Biol 131:655-667.

Feske S, Gwack Y, Prakriya M, Srikanth S, Puppel S-H, Tanasa B, Hogan PG, Lewis RS, Daly M, Rao A. 2006. A mutation in Orai1 causes immune deficiency by abrogating CRAC channel function. Nature 441:179-185.

Graef IA, Wang F, Charron F, Chen L, Neilson J, Tessier-Lavigne M, Crabtree GR. 2003. Neurotrophins and netrins require calcineurin/NFAT signaling to stimulate outgrowth of embryonic axons. Cell 113:657-670.

Gwack Y, Srikanth S, Feske S, Cruz-Guilloty F, Oh-Hora M, Neems DS, Hogan PG, Rao A. 2007. Biochemical and functional characterization of Orai proteins. J Biol Chem 282:16232-16243.

Gwack Y, Srikanth S, Oh-Hora M, Hogan PG, lamperti ED, Yamashita M, Gelinas C, Neems DS, Sasaki Y, Feske S. 2008. Hair loss and defective T-and B-cell function in mice lacking ORAl1. Mol Cell Biol 28:5209-5222. 
Hill-Eubanks DC, Gomez MF, Stevenson AS, Nelson MT. 2003 NFAT regulation in smooth muscle. Trends Cardiovasc Med 13: $56-62$.

Hogan PG, Chen L, Nardone J, Rao A. 2003. Transcriptional regulation by calcium, calcineurin, and NFAT. Genes Dev 17:2205-2232.

Hubbard MJ. 2000. Calcium transport across the dental enamel epithelium. Crit Rev Oral Biol Med 11:437-466.

Jackson S, Nesbitt W, Kulkarni S. 2003. Signaling events underlying thrombus formation. J thromb haemost 1:1602-1612.

Kawamoto T, Shimizu M. 1990. Changes in the mode of calcium and phosphate transport during rat incisal enamel formation. Calcif Tissue Int 46:406-414.

Lacruz R, Smith C, Kurtz I, Hubbard M, Paine M. 2013. New paradigms on the transport functions of maturation-stage ameloblasts. J Dent Res 92:122-129.

McCarl C-A, Picard C, Khalil S, Kawasaki T, Röther J, Papolos A Kutok J, Hivroz C, Ledeist F, Plogmann K. 2009. ORAl1 deficiency and lack of store-operated $\mathrm{Ca} 2+$ entry cause immunodeficiency, myopathy, and ectodermal dysplasia. J Allergy Clin Immunol 124:1311-1318.e7.

Mercer JC, Dehaven WI, Smyth JT, Wedel B, Boyles RR, Bird GS, Putney JW. 2006. Large store-operated calcium selective currents due to co-expression of Orai1 or Orai2 with the intracellular calcium sensor, Stim1. J Biol Chem 281:24979-24990.

Nanci A. 2007. Ten Cate's oral histology-pageburst on vitalsource: development, structure, and function. Amsterdam: Elsevier Health Sciences.

Prakriya M, Feske S, Gwack Y, Srikanth S, Rao A, Hogan PG 2006. Orai 1 is an essential pore subunit of the CRAC channel. Nature 443:230-233.

Putney JW. 2007. New molecular players in capacitative $\mathrm{Ca} 2+$ entry. J Cell Sci 120:1959-1965.

Robinson C, Hiller CR, Weatherell JA. 1974. Uptake of32P-labelled phosphate into developing rat incisor enamel. Calcif Tissue Res 15:143-152.

Robinson LJ, Mancarella S, Songsawad D, Tourkova IL, Barnett JB, Gill DL, Soboloff J, Blair HC. 2012. Gene disruption of the calcium channel Orai1 results in inhibition of osteoclast and osteoblast differentiation and impairs skeletal development. Lab Invest 92:1071-1083.
Schwarz A, Tutsch E, Ludwig B, Schwarz EC, Stallmach A, Hoth M. 2004. Ca2+ Signaling in identified T-lymphocytes from human intestinal mucosa relation to hyporeactivity, proliferation, and inflammatory bowel disease. J Biol Chem 279:5641-5647.

Simmer JP, Papagerakis P, Smith CE, Fisher DC, Rountrey AN, Zheng L, Hu J-C. 2010. Regulation of dental enamel shape and hardness. J Dent Res 89:1024-1038.

Smith C. 1998. Cellular and chemical events during enamel maturation. Crit Rev Oral Biol Med 9:128-161.

Suga S. 1982. Progressive mineralization pattern of developing enamel during the maturation stage. J Dent Res 1532-1542.

Sui W, Boyd C, Wright JT. 2003. Altered $\mathrm{pH}$ regulation during enamel development in the cystic fibrosis mouse incisor. J Dent Res 82:388-392.

Thompson JL, Mignen O, Shuttleworth TJ. 2009. The Orai1 severe combined immune deficiency mutation and calcium releaseactivated $\mathrm{Ca} 2+$ channel function in the heterozygous condition. J Biol Chem 284:6620-6626.

Vig M, Peinelt C, Beck A, Koomoa D, Rabah D, Koblan-Huberson M, Kraft S, Turner H, Fleig A, Penner R. 2006. CRACM1 is a plasma membrane protein essential for store-operated $\mathrm{Ca} 2+$ entry. Science 312:1220-1223.

Wada T, Nakashima T, Hiroshi N, Penninger JM. 2006. RANKLRANK signaling in osteoclastogenesis and bone disease. Trends Mol Med 12:17-25.

Wang S, Choi M, Richardson A, Reid B, Seymen F, Yildirim M, Tuna E, Gençay K, Simmer J, Hu J. 2014. STIM1 and SLC24A4 Are Critical for Enamel Maturation. J Dent Res 93(suppl):94S-100S.

Yeromin AV, Zhang SL, Jiang W, Yu Y, Safrina O, Cahalan MD. 2006. Molecular identification of the CRAC channel by altered ion selectivity in a mutant of Orai. Nature 443:226-229.

Zhang SL, Yeromin AV, Zhang XH-F, Yu Y, Safrina O, Penna A, Roos J, Stauderman KA, Cahalan MD. 2006. Genome-wide RNAi screen of $\mathrm{Ca} 2+$ influx identifies genes that regulate $\mathrm{Ca} 2+$ release-activated $\mathrm{Ca} 2+$ channel activity. Proc Natl Acad Sci U S A 103:9357-9362.

Zhou Y, Lewis TL, Robinson LJ, Brundage KM, Schafer R, Martin KH, Blair HC, Soboloff J, Barnett JB. 2011. The role of calcium release activated calcium channels in osteoclast differentiation. J Cell Physiol 226:1082-1089. 